\title{
Promotion of Nuclear Medicine-Related Sciences in Developing Countries
}

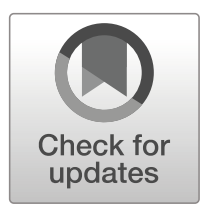

\author{
Dong Soo Lee ${ }^{1,2,3} \cdot$ Yun-Sang Lee ${ }^{1,3} \cdot$ Jae Sung Lee ${ }^{1,3} \cdot$ Min Seok Suh ${ }^{1,2}$
}

Published online: 8 April 2019

(C) Korean Society of Nuclear Medicine 2019

The prosperity of nuclear medicine depends on a variety of factors in many countries. The success of nuclear medicine of the past 2 decades in North America was based on the new instruments developed and installed widely in many institutions, and of course the good availability of ${ }^{99 \mathrm{~m}} \mathrm{Tc}$ and $\left[{ }^{18} \mathrm{~F}\right]$ FDG. Mostly, the wide use of torso PET made the success of 10 million cases of nuclear medicine procedures annually and another 10 million cases of myocardial SPECT. This was equivalent to 6,000 procedures per million. The relationship between the number of clinical volume of nuclear medicine procedures and gross domestic products (GDPs) of the advanced countries are quite proportional in their log-log relationship (Fig. 1). The United States of America (USA) led the era of hybrid imaging mostly of PET/CT, culminating in the introduction of PET/MRI. We think that this evoked great concern among radiologists while they were also pursuing the use of hybrid imaging. The job market in the USA forced nuclear medicine physicians to become half nuclear medicine physicians and half radiologists. There was even a trial integration of the nuclear medicine board and the radiology board, making the nuclear medicine board obsolete. The training system was already mixed between radiology and nuclear medicine in the USA so that 1 year or less would be sufficient to produce radiologists who can do the role of the nuclear medicine physician. Fortunately, after serious discussion among the leaders of nuclear medicine in the USA, the movement of integrating nuclear medicine board training into that

Dong Soo Lee

dsl@snu.ac.kr

1 Nuclear Medicine, College of Medicine, Seoul National University, Seoul, South Korea

2 Molecular Medicine and Biopharmaceutical Sciences, Graduate School of Convergence Science and Technology, Seoul National University, Seoul, South Korea

3 Nuclear Medicine, Seoul National University Hospital, Seoul, South Korea of radiology was given up several years ago. However, the number of new applicants for nuclear medicine board training decreased to two-thirds, job opportunities showed a gradually decreasing tendency, and industry support became reduced. The recent decline of clinical nuclear medicine is related to the new trend of imaging and biomarker developments on a macroscopic time scale. However, the great progress of nuclear medicine during the 1990s and new millennium are derived from the innovative contribution of the best intellects of nuclear medicine physicists and radiopharmaceutical scientists.

In the meantime, developments in the European Union (EU) resulted in pioneering work which is now collectively called theranostics. Theranostics might be considered as the simple combination of therapy and diagnostics; but in more detail, theranostics has the aim to realize personalized medicine using radionuclide therapy and the associated (desirably simultaneous) diagnostic imaging. Here we presume the combination of PET or SPECT imaging concurrently with radionuclide/radiopharmaceutical therapy. I would say that PET/CT, SPECT/CT or PET/MRI should accompany the radionuclide therapy to determine the correct targeting and possible dosimetry providing the effectiveness of the therapy. This can be said to be 'in vivo companion diagnostics' for radionuclide/radiopharmaceutical therapy. The European Association of Nuclear Medicine (EANM) held a pioneering joint symposium around 10 years ago during its annual congress, named the 'International Symposium of Targeted Radiotherapy and Dosimetry (ISTARD),' which was later changed into a 'Do.MoRe' session. This has become the signature session for the annual congress of the EANM in that it heralded the new era of global nuclear medicine, as every regional and country congress now has equivalent or similar sessions of their own. This was also characterized by a collaborative session between therapy, radiopharmaceutical and dosimetry councils. This is small but significant change, since traditionally each council ran the separate and independent sessions in annual congresses. The dosimetry session was sparsely attended, and the therapy session dealt mostly with 


\section{Clinical Volume of NM Procedures vs. GDP 2015}

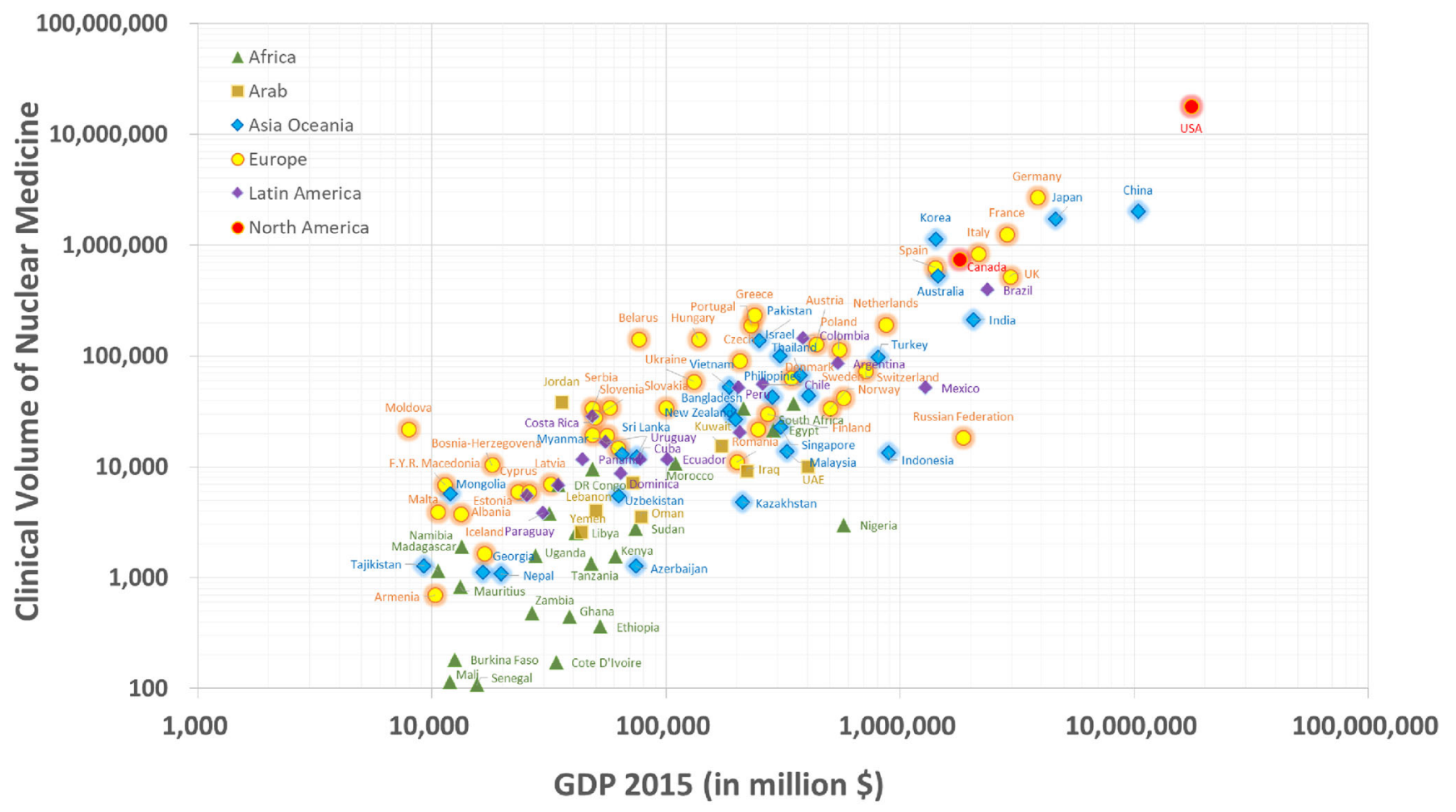

Source: Clinical Volume (NUMDAB IAEA \& DDM2 Report EU, ASNM 2014), GDP (UN)

Fig. 1 Clinical volume of nuclear medicine procedures versus gross domestic product for 2015. The number of clinical volumes is based on the NUMDAB IAEA, 2015 and DDM2 Report EU, 2015 and the documents on the internet. The GDP data from World Bank

the treatment of thyroid cancers. ISTARD achieved a critical mass in the convention and I could feel the earnest exchange of information between participants regarding the current progress and the vision of the near future. The most important thing was to understand what should be done for the progress of therapy or theranostics. The colleagues of the EANM started another endeavor for promotion of theranostics, in which they started the biennial Theranostics World Congress. The spirit of this congress lay in the joint attendance and cross-talk between radiopharmaceutical scientists and nuclear medicine physicians. It has now reached the stage where the participants have started to think about the expansion of the success of theranostics against neuroendocrine tumors and castration-resistant prostate cancers, as well as geographic extension to so-far uninterested countries and the nuclear medicine experts thereof. People need to wait for some time until this collaborative endeavor results in further success for theranostics, i.e. nuclear theranostics with therapy and in vivo companion diagnostics.

In vivo companion diagnostics is analogous to in vitro companion diagnostics, which is well established in the development of new therapeutics such as monoclonal antibodies or kinase inhibitors. In vitro companion diagnostics needs understanding of the surface receptors of cancer cells, the biology of the response of cancer cells for the development of novel therapeutics candidates, and also the way to find new targets and their ligands. This can be done with comprehensive knowledge and/or a library of candidate small molecules, or the innovative expansion of interest in new carriers such as nanomaterials, graphenes or extracellular vesicles. This also mandates the combination of an in-depth knowledge of chemistry, chemical engineering, pharmaceutics, biopharmaceutics, and also nanotechnology. In vivo companion diagnostics needs understanding of the methods to label targeting ligand candidates such as monoclonal antibodies, peptides, aptamers or other newly reported bio-macromolecules with radionuclides using chelators, and of not only the receptor-ligand interaction or binding but also the biological function of the cells after this binding. This is the area of radiopharmaceutical sciences that is mostly chemistry. Later on, when successful, this will move on to radiopharmacy. In vivo companion diagnostics also mandates to adopt molecular imaging instruments such as PET or SPECT. The dosimetry using these instruments, or imaging apparatus, needs physics' help. Acquisition of the information regarding the biodistribution of the radionuclide-labeled novel therapeutics is the prerequisite. Sometimes people acquire the SPECT images (Lu-177, Re-188) or the PET images (Y-90) or even Bremsstrahlung 
images (Y-90). For alpha emitters, we need to develop the alternatives to image the whereabouts of the administered novel therapeutics labeled with alpha-emitting radionuclide. Dosimetry is the ultimate platform upon which we can understand the real doses administered to the targets. In the case of tumors as targets, one needs to set an appropriate minimum absorbed radiation dose to induce their elimination, or in case of the brain targets, one needs to make the novel therapeutics pass through the blood-brain barrier and reach the real target-sometimes abnormal protein aggregates, inflammatory microglia or activated astrocytes. Tracer kinetics is the correct method to let us understand the basic distribution and time course. Consideration must be made of beta-emitters with different energies, consequential Betamax ranges of their own and their characteristic half-lives - both physical and biological. Using this information, the structurally detailed bodily images, and information regarding biodistribution, one now can proceed to calculate the doses to the target organs and the critical normal organs. In vivo companion diagnostics is actually the core theme of nuclear medicine associated with therapy, either classical or novel.

Non-communicable diseases (NCDs), known as chronic disease, claim more than $65 \%$ of all deaths worldwide. NCDs tend to be of long duration and are the result of a combination of genetic, physiological, environmental and behavioral factors. Common NCDs are cardiovascular diseases, cancer, and neurologic disorders. With the rapid increase in NCD-related deaths in developing countries, NCDs are now the major cause of deaths and disease burden in these regions. Especially, based on average life expectancy and per capita national income, developing countries are now transitioning from infectious and communicable diseases to NCDs (Fig. 2). As a consequence, the World Health Organization (WHO) changed its millennium development goal from 2001 to 2015 to the 2016-2030 sustainable development goal. The aim of this agenda was to not only eradicate infectious diseases but also to reduce premature mortality caused by NCDs by one-third.

Nuclear medicine practice, with theranostics and in vivo companion diagnostics, is one of the most important and efficient methods for fighting NCDs. However, the quality and quantity of procedures differ widely from country to country. Difference in economic status, technical advance, human infrastructure and health system policies are some general reasons for such disparity. But there are also some unique problems that nuclear medicine is facing. First, due to constantly and rapidly emerging therapies and techniques, continuous education is essential in nuclear medicine more than in many other clinical fields. In developing countries, the health benefit of nuclear medicine is limited to the large cities or wealthy people. This problem is not because people in these countries cannot afford to pay for the nuclear medicine practice, nor are they without any personnel or instrument/facility infrastructures. In the last decade, these countries have equipped themselves with state-of-the-art nuclear medicine instruments as nuclear medicine practice has become a standard procedure in their healthcare system. However, all the healthcare personnel in these countries were trained in the past environment, when these new instruments were unavailable. Moreover, there are even countries where the nuclear medicine discipline is not taught in the medical course. Second, compared to other clinical fields, research is as important as clinical practice in nuclear medicine. Since the trend of global disease burden toward NCDs has rapidly emerged, developing countries have had a lack of time to pile up clinical cases. Though these countries are equipped with the facilities, infrastructure, and governance of nuclear medicine, they lack the experience and updating opportunities of not only nuclear medicine practice but also nuclear medicine research - either clinical or basic. However, it is not a problem specific to developing countries. Since, the distribution of disease varies by region, country and race, the strengths and weakness in the field of clinical research differ depending on the region, country, and race. Last but not least, nuclear medicine, which deals closely with basic biology, pharmacology, chemistry, and physics in combination, has a multidisciplinary nature. No matter how well-trained a nuclear physician is, it is difficult to perform good clinical practice without collaborating with radiopharmaceutical scientists and nuclear medicine physicists.

Let us imagine that there is a clinical problem bothering the people of a country where there is a nuclear medicine physician. That clinical problem could be the same as found in the advanced countries, such as triple-negative breast cancer or small cell lung cancer with certain genotypes, or could be different from in advanced countries, or unique to his/her particular country, such as a highly prevalent stomach cancer or moyamoya disease, which is a chronic cerebrovascular disease of the internal carotid and cerebral arteries. One needs to adopt from the advanced countries or develop on his/her own from scratch. Nuclear medicine physicians recognize the clinical problems first and then set the goal of establishing the remedy to this problem. For example, he or she builds the platform to treat advanced stomach cancer where the initial presentation of the ulcerative mass was taken care of by surgery and adjuvant chemotherapy with or without the following targeted therapy with kinase inhibitors. However, the most serious problem lies in the metastasis and the driver mutation in the primary or metastatic tumor. There are few precedents for proper treatments for these patients in the advanced countries and the oncologists and nuclear medicine physicians cannot help observing with the feeling that their hands tied. This situation should be overcome by proactively envisioning the future of the country in terms of disease prevalence, people's suffering due to these prevalent diseases and the socioeconomic wish of the patients and their family for recovery of 


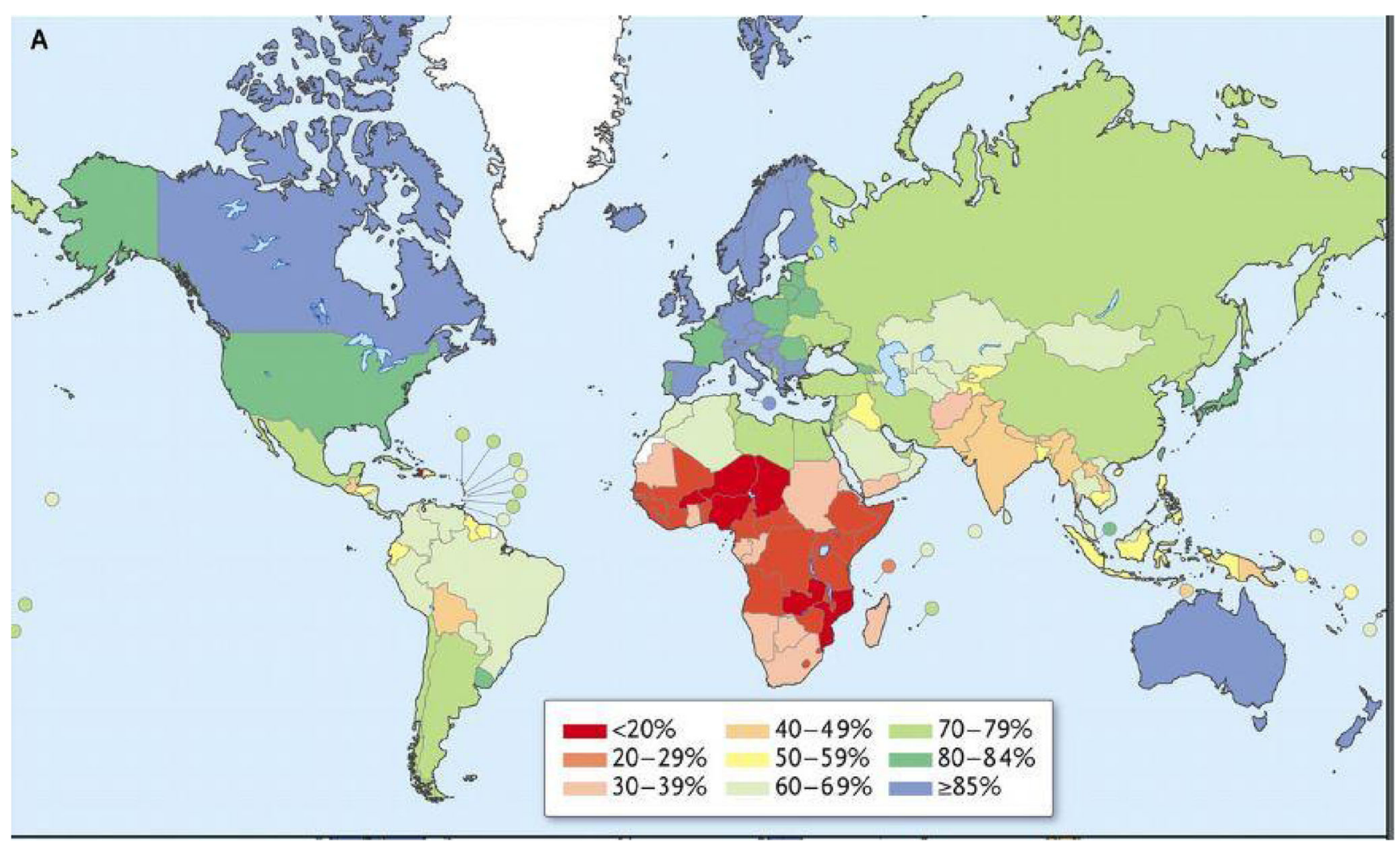

Fig. 2 Percent of non-communicable diseases among the global burden of diseases. In most of Latin America, North Africa, the Middle East, Southeast Asia, and East Asia, the substantial majority of the burden was from non-communicable diseases. Even in South Asia, the fraction due to non-communicable diseases was more than $40 \%$. (With permission from the publisher. Murray CJL, Lopez AD. N Engl J Med 2013; 369:448-457) quality of life. Can nuclear medicine physicians help the surgeons and medical oncologists for this matter? Surely, they can; however, they alone cannot supply the necessary help to the clinicians. Once the problem is recognized, the urgent needs are directed to the well-established radiopharmaceutical scientists and nuclear medicine physicists. The preparation of the team within the premises of nuclear medicine therapy and theranostics needs time, but above all, the enlightenment within themselves about the necessity of these experts and motives rendered by the nuclear medicine physicians and their clinician colleagues, especially in developing institutions, like all institutions/hospitals in the developing countries.

Cultivating the required expertise from scratch is not an easy task. For developing countries, establishment of the needs is almost not recognized by any stakeholders in charge of treating patients. This requirement of the breed of scientists is not so obvious at first and even continuously since then. Nuclear medicine physicians in developing institutions in the developing countries do not have physicists and radiopharmaceutical scientists in his/her department or in the entire nation. Only when they visit institutions in the leading countries do they find experts of that kind and are amazed to see their collaboration. 'Wow, this is nuclear medicine; but in my institution back in my home country, I miss such help from scientists, but I am inspired to build this collaborative environment.' This is the expected sentiment, and this was actually the sentiment of one of the authors, Dong Soo Lee, back in 1993-1994. If we take an example of Korea or my institution, the foresight by the founder of nuclear medicine in Korea made faculty slots for scientists in the hospital/university 2 decades ago. These jobs attracted the best candidates from the fields of pharmacy/chemistry and physics/engineering. Raising the first breed was not without difficulty or failure, but after 20 years they came to be some of the world's best chemists and physicists. They were found to be much more important than the technologists in my institution and in Korea. This might seem to be rare good fortune; however, considering the size of the population of the developing countries, the choice and making the next generation of scientists should be successful ultimately. In this respect, China is also gaining momentum, not mostly from institutions but from industry. How could we expect there to be ten instrument production companies in China a decade ago? No, not at all then. Nine hundred million people in Africa, 600 million in Latin America, and most importantly, around 30 million in Asia and, excluding India and China, we still have 600 million people that are the resources. One can say that previously a country or institution needed quite a sizeable of budget to 
install PET, SPECT, cyclotron, and gamma camera facilities. But recently when we emphasize theranostics, and at least CT and MRI have already been adopted by developing countries for routine clinical practice and considering that the developing countries want badly to decrease the premature mortality by NCD until 2030, we could just introduce SPECT and PET and pay more attention to the practice of theranostics. What we need is just the scenario of small volume and definite treatment which is personalized for each patient. Once surgeons have saved the patient by removing primary mass and adjuvant treatments by radiation oncologists and medical oncologists, the patients are going to suffer from recurrence and metastasis. If we do not contribute to treat these advanced grave patients in a multidisciplinary team, the goal to decrease mortality and increase quality of life is in vain. A pessimistic and/or realistic challenge from a would-be devil's advocate might comment that gravely advanced patients die or suffer in advanced countries too. Yes, that is correct, but it is the reason why we need to incubate and raise the nuclear medicine and nuclear medicine sciences in developing countries. Almost six-sevenths of world's people are living in developing countries, and if nuclear medicine is a real discipline resulting in clinically better outcomes, we should expand the frontiers to fight against and eradicate people's suffering in everywhere in the world. If we need superb colleagues for collaboration in our institutions in the advanced countries, then the discipline of nuclear medicine or the nuclear medicine physicians need highly intelligent scientists around them. In our institution at Seoul National University in Korea, we have ten faculty members, half of whom are scientists (PhDs). In the Radiology Department, they have around 100 faculty and a few scientists, but their performance and the level of academic achievements are already beyond the world-class level. I think this is characteristic of nuclear medicine. This was the case in the era of hybrid imaging in nuclear medicine, i.e. the last 20 years. But this will be much more the case in the coming era of theranostics and therapy.

Dong Soo Lee, a nuclear medicine physician, Yun-Sang Lee, a radiopharmaceutical scientist, and Jae Sung Lee, a nuclear medicine physicist, brought up this issue and the discussion reached the conclusion that it would be better to make a template for producing nuclear medicine scientists in developing countries. For this, first, we collected the experience or wisdom that we acquired during our earlier careers. In the beginning, Dong Soo Lee wanted to be a nuclear medicine physicist, then a radiochemist, and finally a neuroscientist or nanomedicine expert. When he had the good fortune to meet the faculty scientists, Y.S. Lee and J.S. Lee, he no longer needed to aspire to be a physicist or chemist. He still would like to be a neuroscientist, or applied mathematician, or nanotechnologist, but his real identity is a 'science interpreter' to his juniors or prospective medical doctors, and also as a 'nuclear medicine interpreter' to his colleague clinicians. We interpret patients to the clinicians so that they can take clear and better care of their patients within their specialized disciplines. Regarding molecular imaging, after more than a decade of devotion, he found that preclinical molecular biology-based imaging will never be translated to the clinical entity and had better belong to the basic research to be directed to the high-risk small chance innovation for disruptive innovation. However, molecular imaging is the confirmation of pharmacodynamics and if we develop good and successful theranostic radiopharmaceuticals, we should do molecular biology-based imaging for confirmation of the preclinical in vivo companion diagnostics. Just like organoid or patientderived xenograft studies, these preclinical mechanistic studies will be the basis for the promising in vivo companion diagnostics.

Beyond cancer and hybrid imaging and theranostics, I will take moyamoya disease as an example. The pioneering leaders in the advanced countries developed acetazolamide brain perfusion SPECT studies, but the USA and EU have given up on the use of brain perfusion studies. I wonder why, but the USA preferred brain receptor/transmitter PET studies and the EU moved on to heart and tumor studies. Still, the guideline of 2 decades ago recommend a 2-day protocol and allowed the use of dual-head SPECT. Disappointingly, industry followed the trend of these advanced parts of the world and they stopped manufacturing a triple-head SPECT. This was a disaster as we, with the help of earnest technologist colleagues and Jae Sung Lee's devotion, undertook a sincere investigation for an entire 2 years to replace triple-head SPECT with an optimized acquisition mode for dual-head SPECT but were unsuccessful. We are keeping the Trionix camera and are proud to say that we might be one of the rare institutions capable of assessing the subtle impairment of the perfusion reserve of the anterior cerebral artery before or even after revascularization surgery (Fig. 3). We could evaluate the effect of encephalo-galeo-synangiosis surgery to deliver blood flow to the medial frontal lobes of patients with moyamoya disease. If nuclear physicians in the developing countries are confronted with this kind of problem, and fortunately have the best neurosurgeon colleagues in his/ her institution, but no idea regarding how to adopt and optimize the imaging and analysis, perfusion MRI with much lower sensitivity and inaccuracy would have long been used, with harm to the patients, mostly children. Much worse is that they do not even know that they are doing wrong without doing acetazolamide brain perfusion SPECT, and that they do wrong with dual-head SPECT instead of triple-head SPECT and really vague images acquired separately in 2 days and thus not-well aligned. A colleague scientist, in this case a physicist, is indispensable.

We are now pursuing various directions in our research, Dong Soo Lee is continuing to pursue the strict formulation of a topological interpretation of brain connectivity on PET and resting fMRI on PET/MRI and would like to complete the 
Fig. 3 The comparison of perfusion SPECT images acquired using triple-head SPECT and dual-head SPECT. a Triple-head SPECT was acquired with clinically established routine parameters for Tc-99m HMPAO SPECT. b For dual-head SPECT, parameters including zoom factors were optimized to the best after a large amount of trial and error. Shoulder hindered the access of the detectors of dualhead SPECT

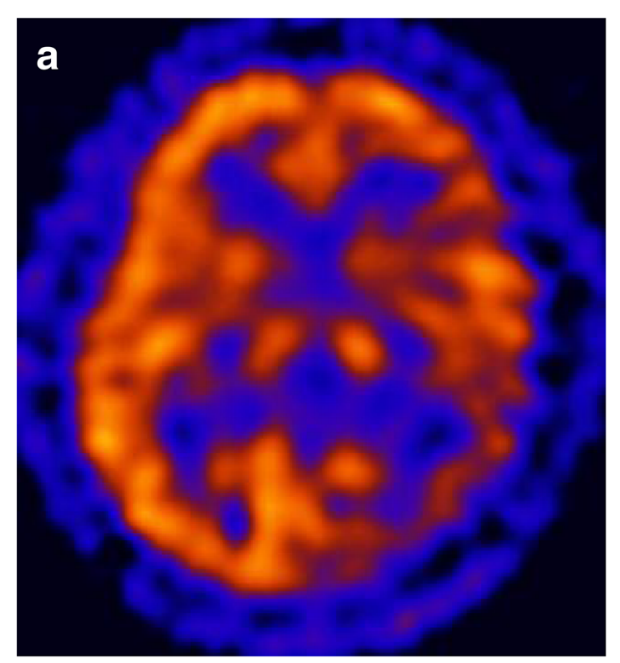

protocol and analysis method for personal brain PET connectomics so that it can finally be used in the clinic. Jae Sung Lee has already made an MR-compatible PET system and launched a commercial animal digital PET/MRI machine. Yun-Sang Lee has been involved in adopting nanomaterials for possible clinical use using Jeong's encapsulation method with multiplexing capability and biorthogonal click reaction that he introduced and now standardization of evaluating the efficacy and toxicity on International Standard Organization (ISO). Though we are doing this high-level research these days, just like colleagues in advanced institutions in the advanced countries, we are the clinically oriented researchers deep to our bones. We agreed that we summarize and explain what we have done for clinical performance improvement during our careers and then we agreed that these explanations would help the next generation of leaders in their developing countries. This information will be made public on the World Federation of Nuclear Medicine and Biology portal web page (http://wfnmbportal.org/bbs/board.php?bo_table=Education) with the privilege of common access for everyone. We will explain our contents as below.

\section{Clinically Raised Problems Dealt with by Sciences in Nuclear Medicine}

This section was contributed by Dong Soo Lee.

Interestingly, nuclear medicine was in charge of the interpretation of clinical problems which should have been explained by physiology and pharmacology. Nowadays this charge has expanded to molecular and cellular biology, and even omics and pharmacology, in which endeavor nothing is beyond nuclear medicine. Once a new phenomenon was discovered-for example, new neurobiological facts such as (1) glutamate/glutamine cycling and the contribution of astrocytes as well as neurons to the amount of flux of glucose consumption, or (2) new roles of microRNAs or extracellular vesicles in the carcinogenesis or metastasis of cancers making pre-metastatic niches and organotropism (rectal cancers metastasizing to liver and the lungs, breast cancers or prostate cancers metastasizing to bones, or stomach cancers metastasizing to livers and then the lungs of bones)-nuclear medicine would aim at visualizing it while using trace amounts of radiopharmaceuticals and labeled radionuclides. Physiological parameters and blood flow (perfusion) or stress-induced perfusion reserve were successfully measured to make parametric images of the heart and brain. Coronary artery disease or cerebrovascular diseases could be analyzed and interpreted in an immediately cognizable way to let the physiological imaging be used in the clinical routine. In the USA, almost half of the entire clinical volume of nuclear medicine was performed as myocardial perfusion SPECT in 10 million cases annually at its peak several years ago. Gated contrast-enhanced stress cardiac MRI or acetazolamidestress perfusion brain MRI were recently reported to present almost similar images to rest/stress myocardial perfusion SPECT or rest/acetazolamide brain perfusion SPECT, respectively. However, these MRI studies need grams of Gd-contrast agents in contrast to SPECT using micrograms of radionuclide-labeled radiopharmaceuticals.

The catch-up of MRI with PET or SPECT in the assessment of physiologic parameters has raised hope for radiology and concern for nuclear medicine; however, stress studies with their inherent risks are blocking the widespread clinical use of these non-nuclear medicine procedures. Instead, classical structural imaging for visualizing coronary obstruction or stenosis with $\mathrm{CT}$ coronary angiography was able to replace the initial evaluation of the patients with low or intermediate likelihoods of coronary artery diseases but with a great burden of radiation hazard to those patients.

In contrast to these changes, the proactive response of nuclear medicine to the clinical demands moved on to adopt 
molecular biology, cell biology, neuroscience, and pharmacology and the recent exponential growth of knowledge thereof. Almost everything is being re-interpreted every week by the development of these fields and especially the molecular biology materialized molecular and cellular imaging in cells and small animals by influencing the imaging strategies using markers and reporters or by supplying stem cells either engineered or cultured mandating the tracking in vivo. However, this molecular imaging, if associated with optical probes currently, are limited to preclinical studies-i.e., being done in small animals and not in humans - and are optimal for evaluation of the pharmacodynamics. Tracer kinetic interpretation has been enlightening the investigators at first to let them understand the pharmacokinetics of the administered radiolabeled tracers. This capability enabled the evaluation of the newly developed radiopharmaceuticals for their biodistribution, excretion, and metabolism in some, and finally, voxel-based dosimetry for therapeutic or theranostic radiopharmaceuticals.

As theranostic upturn of nuclear medicine has been heralded mostly by European pioneer nuclear medicine physicians and has permeated to the wider world, including Latin America, Africa, Eastern/Central and Balkan Europe, Arab and Asia, the nuclear medicine-related sciences should reinvent their roles in clinical nuclear medicine. Molecular imaging is now solidly implemented as preclinical studies to reveal the pharmacodynamics of novel radiopharmaceuticals in animals and it is going to facilitate the development of new agents. Tracer kinetic physics is to be applied to analyze novel theranostic radiopharmaceuticals to unravel dosimetry-based biological effects of radiation in the framework of molecular internal radiotherapy. Nuclear theranostics should have been applied to every current biopharmaceutical, including monoclonal antibodies such as rituximab, or more recently pembrolizumab, or imatinib, and similar small molecule kinase inhibitors. We called this endeavor 'in vivo companion diagnostics' and this is actually based on the theme of classical nuclear medicine under the tracer principle. While developing the therapeutic biopharmaceuticals, global companies avoided using the discipline of nuclear medicine to find the right position for their new biopharmaceutical products to be used only for the indicated patients and instead tried to overcome the regulatory bottlenecks so as to make their new products become blockbusters. Blockbuster means almost always that many patients do not benefit from the novel drugs. The most recent example is the monoclonal antibodies for immune checkpoint therapy, and it is not surprising that the remission rate is $20 \%$ or less in general for melanoma and others. There has been a very fierce dispute in using 'in vitro companion diagnostics.' In addition to the custom of development by profit-seeking companies, the lack of optimization of indication of novel therapeutics was also because the nuclear medicine community fell short of supplying the necessary technology and methodology. Furthermore, the nuclear medicine community was at a nadir in not developing novel therapeutics by itself and being deeply occupied in just hybrid imaging as the only routine procedure for diagnostic purposes. The investigators' interests and the capabilities acted together to prevent the appropriate use of nuclear medicine in modern therapy and theranostics, and thus the nuclear medicine leaders in the advanced countries guided wrongly toward diagnostic imaging with advanced and costly instruments without emphasizing theranostics. To the relief of these concerns, they now start to emphasize personalized medicine, individualized medicine and precision medicine following the lead of the future biology-based medicine. This included stem cell therapy, which is evolving now to cell component therapy or even extracellular vesicle therapy, and multi-omics covering all the transcriptomes, epitranscriptomes, genomics, and epigenomics and also the systems biology interpretation for promoting in vitro diagnostics to the extreme. There seems to be a huge gap between the spirits of development of blockbuster drugs and making multi-omics in vitro assay from laboratory-developed test (LDT) to in vitro diagnostics (IVD). This logic of making new therapeutics and its clinical indications (and of course contraindications elucidated) is based upon the well-developed industry of private pharmaceutical companies, which is lacking in developing countries.

Clinicians in developing countries implicitly recognize this paradox and used to have been frustrated, sometimes giving up and surrendering to the situation of his/her country; however, there are people suffering from NCDs and an aging population gradually prone to becoming afflicted with diseases for which the intervention with novel therapeutics is life-saving in some patients but costly and even harmful in many others. I maintain my opinion that nuclear medicine physicians in developing countries should and can transform themselves successfully to understand the academic disciplines underlying this situation, i.e., nuclear medicine-related sciences to overcome this dilemma. We Korean nuclear medicine physicians were in the same situation decades ago and needed to try to lift ourselves up to the global level of problem-solving tasks and our first step was to understand the problems of our country's keenest interest. Luckily with the accompanying economic growth, we could reach the current level of clinical performance of nuclear medicine practice (1 million annually) and the quality of clinical and basic research in nuclear medicine recently. Though there is much room for improvement, we can say clearly two things: one is that we Koreans during the two decades till now were absorbed in pushing forward of advanced hybrid imaging, and the other is that we proactively formulated the manpower for nuclear medicine-related sciences and the organizations composed by our scientific expert colleagues. The became the Korean Council of Nuclear Medicine Imaging and Instruments and the Korean Society of Radiopharmaceuticals and Molecular Probes. 
Theranostics or therapy in nuclear medicine really need to expand the discovery of the possible resources finally being used as radiopharmaceuticals. Ever-changing and newlydeveloped therapeutics in cancer and other NCDs need to involve nuclear medicine imaging as 'in vivo companion diagnostics.' This is really the way nuclear medicine is moving forward in the upcoming years ahead of nuclear medicine physicians regardless of whether we belong to the advanced countries or the developing countries. The advantage of nuclear medicine therapy over hybrid imaging, such as PET/ MRI, resides in the fact that we need only the correct dose individualized to the patients. The pioneering leaders of the well-resourced institutions are strongly required to elaborate the optimal doses and simplify the follow-up (imaging) methods with SPECT or PET based on well-designed clinical trials, which will generate the evidence required for evidencebased medicine or find the appropriate use of new therapeutics. The clinical application of nuclear theranostics should start almost synchronously not heterochronously all over the globe if it wants to be an essential medicine. Making the clinical nuclear medicine affordable should start, strangely and interestingly, by creating the nuclear medicine-related science infrastructure in the developing countries. Nuclear medicine-related sciences can prosper in countries having a large population, such as China, India, Bangladesh, Pakistan, Indonesia, and Vietnam - if I indicate the countries having a population over 100 million. The best intellects, like my colleagues here, Y.-S. Lee and J.S. Lee, in this commentary article are an example for every developing country, saying that they also can raise the next-generation leader scientists in their country in addition to the currently leading scientists. This will bring out the new era of nuclear medicine in the coming better economy of these countries, which will soon be realized by each country and by all their people's efforts.

The materials we used to explain in detail the path we trod regarding our implementation of nuclear medicine-related sciences during a 4-day visiting workshop in Dhaka, Bangladesh during December 8-12, 2018 have been uploaded to http:// www.wfnmbportal.org/bbs/board.php?bo_table=Education and can be downloaded with the principle of Creative Commons.

\section{Radiopharmaceutical Chemistry}

This section was contributed by Yun-Sang Lee.

Radiopharmaceuticals possess typical features, such as a short half-life, little or no pharmacological effect from chemical composition, and the spatial limitation at the specially equipped and licensed area for radiation protection, which differ from conventional medicine. Therefore, radiopharmaceuticals should be handled with optimal caution and prepared shortly, and sometimes, the full scale of quality control (QC) is not possible before their clinical use. Because of the risk of radiation exposure and chemical or biological contamination of the final product in the radiopharmaceutical preparation, we need to combine the expertise of rapid pharmaceutical preparation and the proper handling skill of radioactive materials.

In the lecture in the visiting workshop, we focused on how to set-up the radiopharmaceutical preparation, especially in a newly established nuclear medicine department with limited man-power and equipment. Even though still the current situation of each nuclear medicine department in the developing countries is not characterized, we can start the easy-to-use radiopharmaceuticals, such as compounding of ${ }^{99 \mathrm{~m}} \mathrm{Tc}$ radiopharmaceuticals, and widen the PET and therapeutic radiopharmaceuticals. I started my career in radiopharmaceutical sciences 20 years ago in Seoul National University Hospital as a research scientist under the guidance of Prof. Jae Min Jeong, who is the current President of the Society of Radiopharmaceutical Sciences (SRS). At that time, we tried to develop a new radiopharmaceutical for a myocardial imaging agent and a therapeutic radiopharmaceutical with limited manpower and equipment for organic synthesis and radiochemistry. After the struggles of my $\mathrm{PhD}$ thesis, I realized that my mission would be the fast adoption of new radiopharmaceuticals to the clinic, which would be manufactured products or only available for radiopharmaceutical compounding. After 10 years of effort by myself and our cyclotron technologists, we can now use four MFDS (Ministry of Food and Drug Safety)-approved manufactured radiopharmaceuticals $\left(\left[{ }^{18} \mathrm{~F}\right]-\mathrm{FDG},\left[{ }^{18} \mathrm{~F}\right] \mathrm{NaF}\right.$, $\left[{ }^{18} \mathrm{~F}\right] \mathrm{FDOPA}$ and $\left.\left[{ }^{13} \mathrm{~N}\right] \mathrm{ammonia}\right)$ and nine compounding products in the clinical routine. Moreover, we can use more than 30 PET probes for clinical research.

In the course of the visiting workshop, therefore, we start from understanding of the special features of radiopharmaceuticals, and production of radionuclides. Then we touch the preparation of ${ }^{99 \mathrm{~m}} \mathrm{Tc}$ radiopharmaceutical, therapeutic and generator-based radiopharmaceuticals, and PET radiopharmaceuticals. Finally, we discuss the current status of regulatory issues of the use of radiopharmaceuticals.

During the preparation of this course, I found that we have very little information from radiochemists, technologists and radiochemistry facilities, especially in developing countries. So, we need to gather the basic information about the current situation of radiopharmaceutical chemistry, the number of cyclotrons, radionuclide and radiopharmaceutical production facilities, manpower, etc. of each country by the global body of this field, such as 
The World Federation of Nuclear Medicine and Biology (WFNMB) or The Society of Radiopharmaceutical Sciences (SRS). This will be sure to facilitate the propagation or the best and proactive radiopharmaceutical sciences to be applicable to the clinical nuclear medicine in the start-up departments in the developing countries.

\section{Advances in Physics, Instrumentation, and Dosimetry}

This section was contributed by Jae Sung Lee.

The physics lectures in this course cover various physics and engineering related topics in nuclear medicine, from imaging principles to recent advances. These topics include the basic physical principles in nuclear medicine imaging devices, such as PET and SPECT. During the past decade, we witnessed many remarkable technical advances in nuclear medicine and related hybrid imaging systems. One of the key advances in those imaging systems is the evolution of PET and SPECT detectors. In PET, the silicon photomultiplier (SiPM), a semiconductor photosensor with high signal amplification gain and compact size, is replacing the conventional photomultiplier tubes (PMT). All the major PET/CT vendors offer digital PET systems based on the SiPM with much better system performance than the PMT-based PET systems. The compactness of SiPM enables the better scintillation light collection that leads to the improvement in timing resolution of recent clinical PET systems and insensitiveness to the magnetic field change allows this new photosensor to be widely used in several clinical and preclinical PET/MRI systems. Improving the timing resolution of the BGO PET detectors using ultraviolet SiPM is an interesting approach, particularly for developing countries, because BGO is much cheaper than LSO or LYSO. In SPECT, cadmium zinc telluride (CZT)-based cardiac dedicated systems allow faster SPECT scans or lower radiation dose. Further advances in CZT detector and collimator technologies have led to the improvement in sensitivity of CZT SPECT systems. Much smaller dead space in CZT detectors than a conventional Anger camera offers better flexibility in SPECT system design. The CT-derived attenuation map in SPECT/CT and collimator-detector response-based image reconstruction algorithms have dramatically improved the quantitative accuracy of SPECT images. In addition, artificial intelligence and machine learning technologies continue to pave their way into nuclear medicine. Some deep learning-based nuclear medicine image generation and restoration algorithms outperform conventional mathematical and statistical approaches.

Although the availability of targeted radionuclide therapy for cancer patients is growing, the radiation toxicity is still the main limitation of this therapeutic approach. Therefore, the importance of personalized dosimetry for safe and effective personalized treatment is emphasized. Although a practical and robust dosimetry method is necessary for the routine use and wide clinical adaptation, the investigators who dedicate themselves to improve the accuracy of dosimetry should be continuously encouraged for us to achieve better treatment outcome and patient safety. SPECT/CT and PET/CT enable more accurate estimation of the time-integrated activity than planar scintigraphy. However, these tomographic methods are less practical than planar scintigraphy because we should acquire sequential data sets for the radiation dose calculation. A hybrid method that combines planar and tomographic imaging data would be a good alternative to the sequential tomographic imaging studies considering the trade-off between accuracy and practicability. Although voxel-based dosimetry based on Monte Carlo (MC) simulation that accounts for heterogeneous activity and medium distribution provides an accurate dosimetry estimate, MC simulation demands extensive computational resources and time. A recent study showed that a less time-consuming multiple voxel S-value approach yielded compatible dosimetry estimates in voxel level to those of direct $\mathrm{MC}$ simulation.

\section{Conclusion}

Developing countries are now facing increased risk of NCDs. We now have the concept of theranostics and in vivo companion diagnostics, which is the key component for nuclear medicine to fight against such a crisis. However, with the efforts of physicians alone, we insist that we cannot move on. Nuclear medicine, which has inherent multidisciplinary nature, can truly develop when collaborating with basic scientists such as nuclear medicine physicists and radiopharmaceutical scientists. We have the tools and methods, and above all the good will, to realize the future. By building up workshop experiences, education materials, and clinical cases, we are developing a web-based e-learning platform, which can also be used as a communication channel for global nuclear medicine society. Using an e-learning platform we can accelerate the continuous propagation of up-to-date basic science achievements, which can be customized to the developing countries and state-of-the art nuclear medicine practice globally over the countries and continents. The first breed of basic scientists in developing countries can adapt themselves to get connected with the rapidly evolving disciplines of theranostics and therapy in nuclear medicine, finally enhancing nuclear medicine to the benefits of humankind, regardless of their country's current socioeconomic status. Or rather, at least the maximal 
benefits from nuclear medicine allowed now and limited by their current level of economic development.

Acknowledgments We thank participants on site in NINMAS (National Institute of Nuclear Medicine and Allied Sciences), Dhaka, Bangladesh and online; Drs. Su Thet Oo of Myanmar, Deverly Tumapon of Philippines, Hendra Budiawan and his colleagues of Indonesia, and Pham Cam Phuong and her colleagues of Vietnam during the visiting workshop supported by KOICA (Korea International Cooperation Agency) and their kind feedback, including questions about the workshop contents presented by the four authors of this comment.

\section{Compliance with Ethical Standards}

Conflict of Interest There is no conflict of interest with any financial organization regarding the material discussed in the manuscript.

Publisher's Note Springer Nature remains neutral with regard to jurisdictional claims in published maps and institutional affiliations. 